and some small amount of secretion. This condition is seen mostly in young children.

Vernal catarrhal conjunctivitis more nearly resembles trachoma, but the peculiar bluish appearance of the conjunctiva without the distinctly enlarged capillary vessels, the peculiar white, frothy, sticky secretion, the violent itching of the lids, and the distinct acute attack in spring and the much more tolerable condition in winter, make up the picture for vernal conjunctivitis.

We divide trachoma into three stages; the follicular, the papillary or hyperplastic, and the cicatricial or connective tissue stage.

Among our school children, we have to do almost entirely with the follicular stage of the disease. Occasionally we see one in the papillary stage. It is unusual to find a case of trachoma in young children, but I have scen a few cases as young as six years, where there were adult cases in the family.

The onset sometimes is very insidious, without any distinct inflammatory process. We find the distinctive, grayish white, semitransparent bodies, that have been called sago-grain granulations. They may be disseminated or in rows. They are mostly confined to the palpebral conjunctiva and the upper retrotarsal fold.

The mucous membrane, that contains the trachoma bodies which fill up the tissues, is pale or yellowish red. Later, as the inflammatory stage progresses with an occasional exacerbation, we find additional discharge that sticks the edges of the lids together. After this stage has been reached, there is no controversy about the diagnosis.

\section{PANOPHTHALMITIS OF EN- DOGENOUS ORIGIN.}

\author{
F. S. CoOK, M.D.
}

EAU CLAIRE, WISCONSIN.

Mrs. S., referred to me August 19, 1920 , complaining of severe pain in right eye of two days duration.

Past history: Had been perfectly healthy up to four days ago, when she had a slight headache, increasing in severity, and localizing in the right eye in the morning of the second day. Began menstruating at this time. The pain in the eye, towards night of the second day, became severe and she put "musterole" on the forehead and eye lids at this time. Pain increased in severity and a doctor was called, who gave her morphin, also used eserin in the eye as he could detect an increased tension. Next day, lids were swollen and eyeball was very hard, pain severe and no vision. I saw her the first time at noon on the third day.

The eye presented the following condition: Lids swollen, conjunctiva injected, movements limited, some exophthalmos, cornea flat, striated and opaque. Infiltration was in the deep layers of the cornea. Tension $60 \mathrm{~mm}$. (Gradle Tonometer). Temperature 102, nose normal, no pus, no evidence of ethmoid disease. $\mathrm{X}$-ray report shows frontal, ethmoid, sphenoid and antra clear. No apical abcess. General examination by an internist reported negative, urine negative, blood count, red cells $4,500,000$, whites 9000 , H. B. $85 \%$. Smears from the eye show micrococcus catarrhalis. She was put in the hospital, eserin gr. 1 to the oz., 1 drop in the eye every hour. Hot packs, 20 minute every hour and mag. sulp., morphin to control pain. Next day, Aug. 20 th, pain was still severe in spite of morphin. There was marked swelling of the lids and exophthalmos was more marked, eye almost stony hard. There were marked lines of pressure on cornea. Eserin was discontinued and hot mag. sulp. stupes put on the eye. At this time the eye seemed to be pushed down and out. White count was 15,000. Deep incisions were made in the orbit under nitrous oxid. No pus could be located. Canthotomy was done. Patient put in a restless day, temperature $1026 / 10^{\circ}$. By night cornea was sloughing. The question of enucleation vs. evisceration was discussed pro and con and enucleation decided on for the reason we had not been able to locate any pus in the orbit and felt reasonably sure it was there.

Operation was performed August 21 st, five days after first symptom. 
Right eye enucleated, tissue very edematous. On incision of Tenon's capsule, a few drops of pus were found at the outer angle. The eye ball was ruptured in two places in freeing adhesions, vitreous escaped. Vitreous fluid, cloudy and looked like pus. Cornea was completely ulcerated. Palpation of orbit, after eye ball was removed, did not disclose any localized pus or thickening of periostium over ethmoid region. Guttapercha drain was put in center, Conjunctiva sutured. Convalesence was uneventful. Temperature dropped to normal on second day after the operation and continued so. On the second day patient complained of pain in the right shoulder, no redness or swelling. This disappeared on the fourth day with no other treatment than heat. Discharged in two weeks.

\section{Pathologic Report by Dr. King, Pathologist.}

Gross Specimen: Cornea hazy, ulcerated,ciliary body covered with fibrinous exudate. Retina greatly thickened and thrown in ridges. Section shows cornea denuded of epithelium. Entire thickness shows massive invasion of polymorphonuclear leucocytes. The ciliary body is thickly sprinkled with polys and red blood cells. The ciliary muscle shows invasion. The ciliary processes are embedded in an exudate of fibrin and polys. The retina shows the same exudate and masses of polys are seen in the choroid and sclera. Hyperemia marked thruout. Bacteriologic findings of exudate. Many chains of streptococci. Diagnosis: Suppurative Panophthalmitis.

\section{Comments}

Here is a case of panophthalmitis of endogenous origin, coming on in a strong, healthy woman, without any apparent foci of infection. The only disturbance was her menstrual period. Could the embolus come from the distended venous sinus of the uterus? We are told this uterus is the most productive place of emboli. The early stages presented the typical picture of acute fulminating glaucoma. The increased pressure, no doubt, was dure to the swelling of the orbital tissue press- ing on the back of the eyeball and the eyeball being held back by the lids, muscles, etc. There was no pus in orbit and no rupture of the globe previous to operation. What was the cause of the cellulitis? Our classics tell us that we get cellulitis in panophthalmitis of endogenous origin, but it is late and after a rupture of the globe has taken place.

Fuchs's tell us that Tenon's capsule may be implicated in inflammation of the eyeball, so that the inflammatory edema develops in the capsule itself and in the adjoining cellular tissue of the orbit, and that the eyeball is thus pushed forward. Hence a slight degree of exophthalmus is sometimes found in severe cases of irido-cyclitis and in panophthalmitis. Could this be due to the secondary invasion of the Tenon's capsule thru the ciliary veins of the globe?

Ball says that inflammation of the choroid by metastases fortunately is of rare occurrence. It has been found during cerebro-spinal meningitis, typhoid fever, scarlet fever, puerperal fever, erysipelas, mumps, caries of the cranial bones, ulcerating endocarditis, septicemia following surgical operations and compound fractures, and in the course of pneumonia due to influenza. The existence of purulent choroiditis as a metastatic affection was established by Virchow in 1856 . Both eyes are usually affected, one after the other. Bull, of New York, who has carefully studied six cases occurring in the course of grippe-pneumonia, and has made two autopsies, states that the microorganisms found in such cases are the staphylococcus albus and aureus, the streptococcus pyogenes, and the pneumococcus. Postpartum metastatic choroiditis usually appears between the sixth and fourteenth days after delivery and is more frequently unilateral than bilateral. It is a sign of the gravest import. Of sixty-three cases collected by Axenfeld, twenty-two were bilateral. Prognosis as to life, in unilateral cases is good; on bilateral, usually fatal. Of nine cases observed by Hirschberg, all died. Recoveries however, have been recorded by Kipp and Wood. Kipp's case was unilateral, and Wood's was bilateral. 
Hansell in the Annals of Ophthalmology of 1912, reports a case coming on during the course of acute inflammatory rheumatism. The patient died at the end of the third day. Cultures from the blood showed streptococci, but none could be found in the eyeball.

Graefe and Saemisch make the statement that it is not always possible to find a localized source of pus; and in such cases, one must assume that the bacteria have entered the body thru a mucous membrane without having excited evident inflammation.

Axenfeld tells us that metastatic ophthalmia is due primarily to the introduction of septic masses into the capillary vessels of the eye. In the binocular form, the first tissue to be infected is the retina; in the monocular, the uvea.

\section{SOCIETY PROCEEDINGS}

Reports for this department should be sent at the earliest date practicable to 1 r. Harry S. Gradle, 22 E. Washington St., Chicago, Illinois. These reports should present briefly the important scientific papers and discussions.

\section{COLLEGE OF PHYSICIANS OF PHILADELPHIA, SECTION ON OPHTHALMOLOGY.}

$$
\text { May 17, } 1921 .
$$

Dr. G. Oram Ring, Chatrman.

Double Coloboma (Nasal and Temporal) of the Optic Nerve.

Dr. B. Alex. Randall reported the history of a young woman, whose left eye, hyperopic with practically normal vision, has shown no change in the 12 years she has been under observation. Instead of a vertically oval disc as on the right, she presents a large horizontally oval disc with deep central porus and absorbing crescents of the choroid at both lateral margins. Within these, defined by no "scleroid ring," each disc margin shows a dark "bottomless" depression, sharp-cut outward: the one to the nasal side having the upper nasal vessels passing under its margin, somewhat as the other upper vessels are related to the steep edge of the porus. There is no trace of a colobomatous gap in the choroid or iris. Vision and refraction are equal in the two eyes.

It is a matter of interest that the grandmother presented to the nasal side of her left eye a horizontally oval lesion of the choroid, that might readily have been mistaken for coloboma. He had seen this, however, in its first stage as a patch of choroiditis, in the center of which a bleb of retina was detached by exudate which ruptured into the vitreous and was absorbed, the vague yellow patch of inflamed choroid then going on to atrophy, with pigment heaping at its margins. The first stage of this condition is beautifully shown in Jaeger's plate, but so far as he knew there is no recorded observation of the later stage, as he had the chance to observe it thru a series of years.

\section{Capsulo-Muscular Advancement With-} out Incision.

Dr. S. Lewis ZIEgLer exhibited a sccond case of his simplified operation to supplement the one shown before the Section about a year ago.

A capsulo-muscular advancement of the external rectus of left eye was performed by suture alone, without incision, which brought the eye almost into position. This was accomplished by grasping the muscle thru the conjunctiva, about $12 \mathrm{~mm}$. back from the cornea, and entering a double armed suture by a whip-stitch, first thru one margin of the muscle and then thru the other. The needles were then carried forward over the conjunctiva in a line parallel with each muscle margin and inserted in the sclera at the junction, deeply enough to secure a firm scleral anchorage. The suture was tied in a double surgical loop, and the tissues drawn forcibly forward until the necessary reposition was secured, when the second loop of the knot was tied. 